\title{
Mathematics Applied to the Study of Bicycles
}

\author{
Jim Papadopoulos
}

A riderless bicycle has remarkable properties of self-stability. If it is knocked sideways when rolling forward on flat pavement, it may briefly wiggle left and right and then return itself to upright straight rolling. This is surprising to most of us, but in fact has been rediscovered countless times by children, and it has been analyzed mathematically by scores of investigators. The behavior is documented online with titles like "Riderless bicycle," "Ghost riding motorcycle," etc.

Such self-stability is not universal, however. The bicycle must be in good shape, it must be symmetric, its dimensions and masses must lie within certain limits, and the speed must be neither too low nor too high.

A question that has inspired me for decades is: When and why is a bicycle self-stable? I originally came to this problem through bicycle racing, having grown skeptical of the bike shop wisdom that very slight variations in bicycle shape could cause dramatic changes in the ease of balance and control by a rider. In 1986 I went to Cornell to look into the problem with Andy Ruina.

Care is needed when formulating the precise question to be studied. For example, there is a feel of stability or of resistance to turning; there is the ability to ride no-hands; and there is the behavior of a riderless bicycle, either with or without $100 \mathrm{~kg}$ extra mass to represent the person. These may be only loosely related.

Anything involving human behavior is potentially very complex. For example, when struck by a wind gust, rider response will depend on skill, fatigue, and the properties of the bicycle. Different riders will respond in different ways. Therefore I have focused on the simple question of whether a given riderless bike is or is not intrinsically stable at a given speed.

Inherent bicycle stability can be investigated either by mathematical analysis of an ideal dynamic system or by

For permission to reprint this article, please contact: reprintpermission@ams.org.

DOI: http://dx.doi.org/10.1090/noti1556

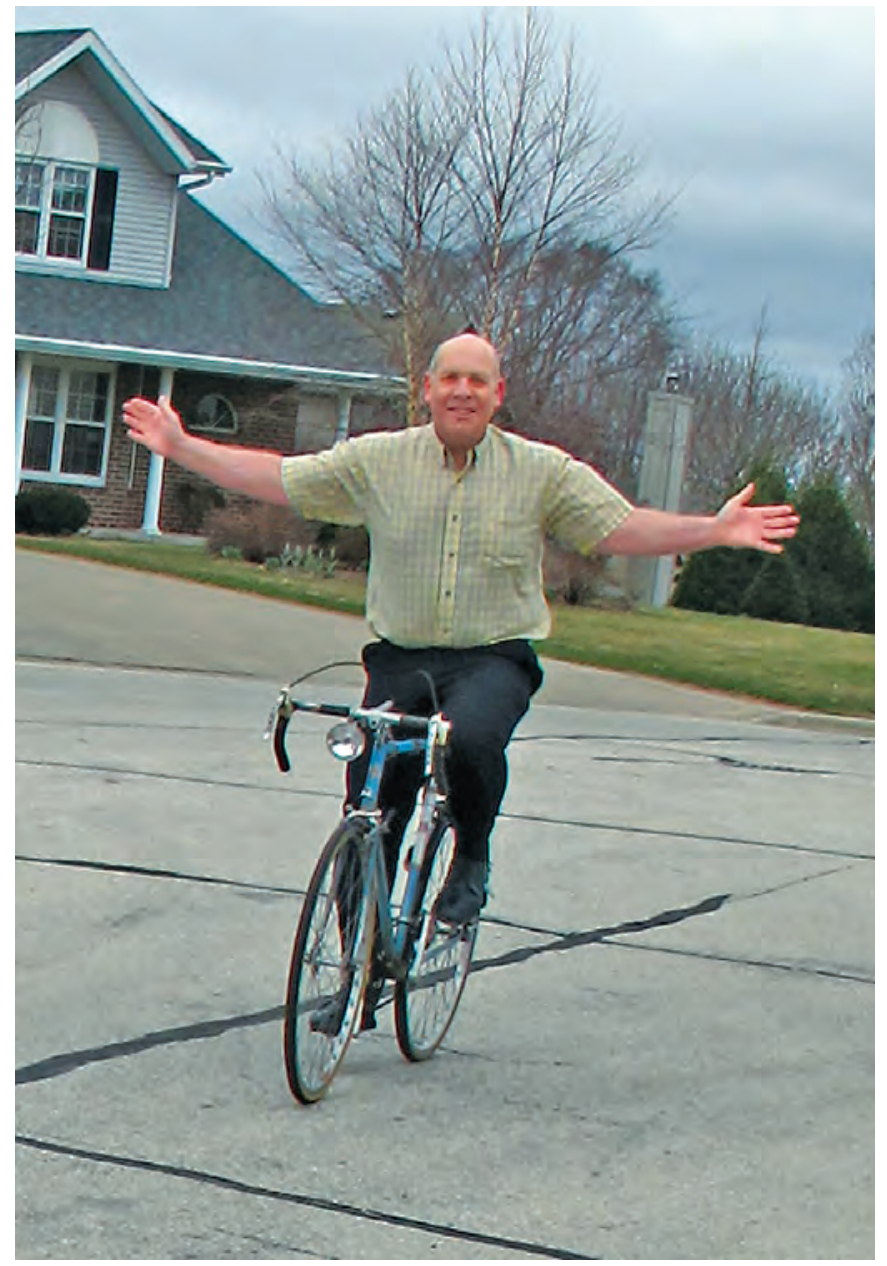

Jim Papadopoulos demonstrates that a bicycle has remarkable properties of self-stability.

Jim Papadopoulos is assistant teaching professor of mechanical and industrial engineering at Northeastern University. His e-mail address is J.Papadopou1os@northeastern. edu. 

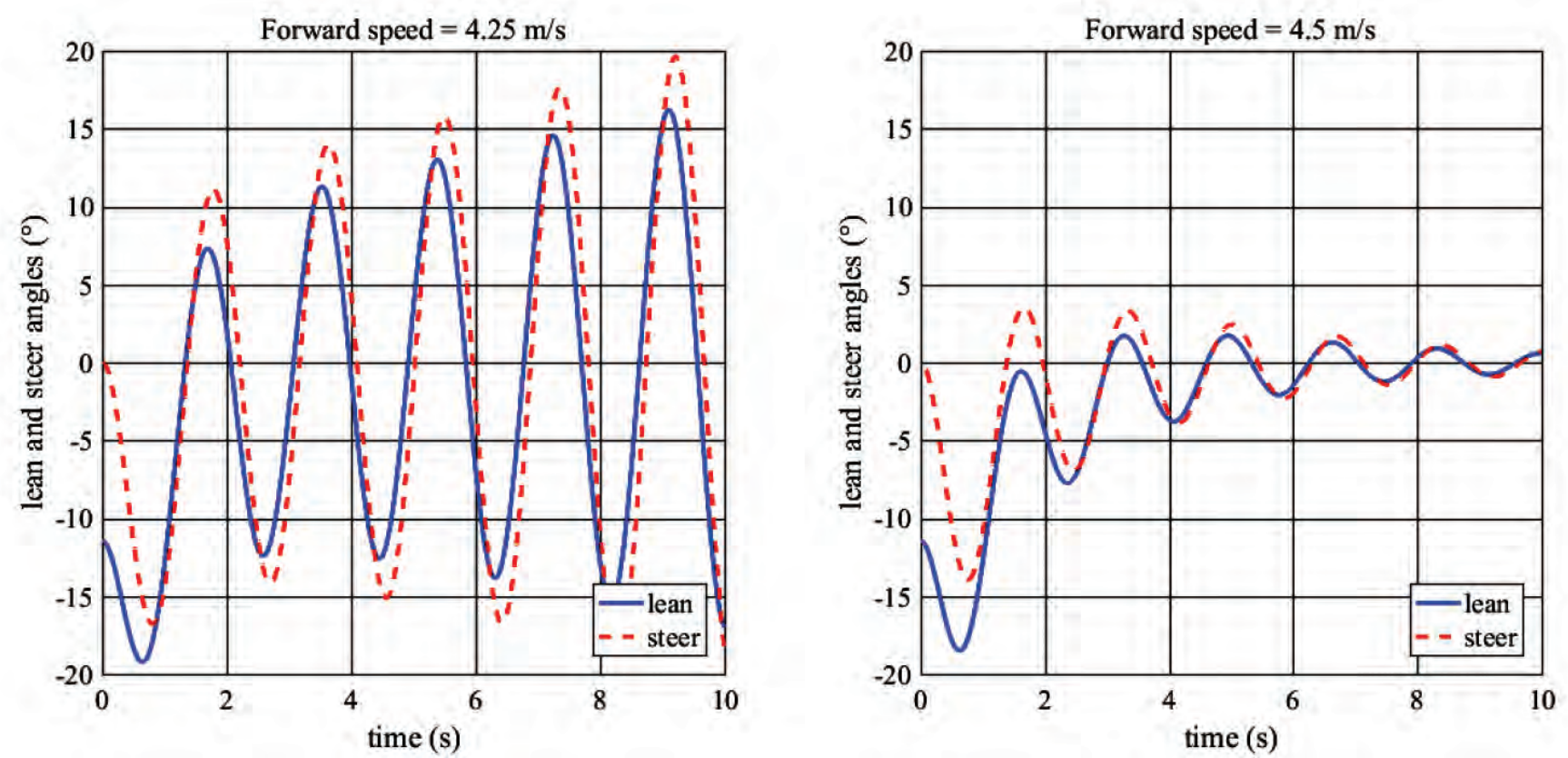

Figure 1. Below the stabilizing speed, the bicycle wobbles more and more, while above the stabilizing speed, the wobbling disappears. Computed evolution of lean and steer angles provided by Andrew Dressel using his MATLAB program, JBike6.

\section{When and why is a bicycle self-stable?}

experiments on actual hardware with all its messy and inconsistent friction and vibration behavior. We chose the former. While it has proven hard for many to get the math right, once that is done the result is solid-for the given ideal system. Thus my focus has been on mathematical analysis of the ideal riderless bicycle, though with the unhappy awareness (a) that the ideal model may have left out physical phenomena that are important to real bicycle behavior and (b) that self-stability is only one part of the riding experience.

Our reported research rests on two foundation stones: - One is the definition of a minimal problem. We stripped the problem down to simple questions of the existence of linearized stability (i.e., the disappearance of small disturbances) for ideal dynamic systems approximating bicycles. We specifically did not look at ease of no-hands control by a rider. We also eliminated many factors, for example, the wiggling of a rider and steer-resisting friction of the flattened tire. Finally, we did not relate the findings to rider preference.

- The other is the care in analysis. Scores of previous investigators made errors in setting up their equations or deducing consequences. We spent a lot of effort to compare our work to previous results and to derive our results in multiple ways that provided confirming matches.
In the work culminating in the 2007 Royal Society publication of "Linearized dynamics equations for the balance and steer of a bicycle: a benchmark and review," we focused entirely on determining the correct mathematical equations to describe a specific system of frame, steering fork, and wheels rolling freely and nearly upright on level ground. (When I say "we," I am referring to principally Andy Ruina and Arend Schwab, who kindly invited me to participate in extending and publishing research initiated at Cornell. Andy also personally supported my involvement financially.)

The result is two coupled second-order differential equations, comparable to those describing two pendulums connected with a spring and a damper (a kind of shock absorber). There are differences in detail, however. While the coupled pendulums come to rest due to energy dissipation in the damper, the energy of the bicycle's lateral motion can either fall or rise (due to interchange between forward kinetic energy and the energy of tipping).

The equations govern the lean angle $L$ of a riderless bike and the steer angle $S$ of its front wheel and handlebars. (For clarity, I have defined $V_{\mathrm{L}}$ as the velocity of the lean angle, that is, the rate of change of angle, and $A_{\mathrm{L}}$ as the acceleration of the lean angle, that is, the rate of change of the velocity. Those who have studied calculus will recognize these as the first and second derivatives of $\mathrm{L}$ with respect to time. Likewise I will use $V_{\mathrm{S}}$ and $A_{\mathrm{S}}$.) They take the following form:

$$
\begin{aligned}
& M_{\mathrm{LL}} A_{\mathrm{L}}+M_{\mathrm{LS}} A_{\mathrm{S}}+C_{\mathrm{LL}} V_{\mathrm{L}}+C_{\mathrm{LS}} V_{\mathrm{S}}+K_{\mathrm{LL}} L+K_{\mathrm{LS}} S=0 ; \\
& M_{\mathrm{SL}} A_{\mathrm{L}}+M_{\mathrm{SS}} A_{\mathrm{S}}+C_{\mathrm{SL}} V_{\mathrm{L}}+C_{\mathrm{SS}} V_{\mathrm{S}}+K_{\mathrm{SL}} L+K_{\mathrm{SS}} S=0 .
\end{aligned}
$$


These equations are the physics consequences of Newton's laws applied to the infinitesimal particles that make up the bicycle parts when in motion while subject to gravity. Each involves the lean acceleration, steer acceleration, lean rate, steer rate, lean angle, and steer angle. The coefficients of $A, V$, and $L$ or $S$ (like $C_{\mathrm{LS}}$ ) are complicated functions of the bicycle dimensions, mass distribution, and velocity.

Given all the bicycle parameters, leaning and steering motion can be determined numerically using Excel or MATLAB. At a specific instant, if the steer and lean rates are known and the steer and lean angles are known, then the two equations can be cast as two easily solved linear equations for lean and steer acceleration in terms of all other quantities. Then the solution proceeds as follows:

- The steer acceleration lets us compute the change in steer rate over a short time.

- In that same time the steer velocity lets us compute the change in steer angle.

- The same steps are possible for the lean angle.

Thus, we have the means to transition from one instant to a slightly later time and will have the information to repeat the computation as many times as desired. Figure 1 shows how a slight change in bicycle speed from 4.25 to 4.5 meters per second leads from instability and growing oscillation in the left plot to stability and shrinking oscillation in the right plot.

With correct equations, one can define a given bicycle in a given configuration and state of motion, then numerically solve the equations to see whether it rights itself. This is a kind of calculational experiment, perhaps of interest to a specific situation, but not revealing any general results about bicycle design.

Potentially much more revealing is a study of the stability of ALL solutions of the given equations. Some great theoretical results from the 1800 s dictate that the system will be stable if products of the equation coefficients (such as $M_{\mathrm{LL}}, K_{\mathrm{SL}}$, etc.) satisfy a number of inequalities called the Routh-Hurwitz criteria.

Using this approach, we were able to investigate a couple of general questions. Among bicycle makers and users, it was universally agreed that stability was only possible if some specific design criteria were met. One was that the wheels should exhibit adequate gyroscopic strength, requiring extra torque to turn. In other words, it was believed that a bicycle on skates could never be self-stable. Another presumed requirement was that the front wheel should touch the ground behind the line of the steering axis, a kind of caster distance.

We theoretically outlined a simplified bicycle shape, and adjusted its dimensions to show that it could satisfy the Routh-Hurwitz criteria (thus achieving stability in forward motion) despite violating the above two criteria.
This demonstrated that the widespread beliefs didn't hold water. Furthermore, we built a physical model of this simplified bike and demonstrated the actual recovery from a bump, proving that the mathematics adequately described reality.

This work does not immediately revolutionize bicycle design, however! For one thing, there is no room for a rider. The fact is that bicycles are generally quite good; all we did is show that in exploring changes, one needn't strictly maintain the caster and gyroscopic components as formerly believed. Furthermore, the work is far from complete. One important need is to develop a mathematical model that works with a rider on the bike. For example, when a heavy person is on a bicycle, the tires are flattened and the original equations don't capture the effects of their friction and flex. In addition, a rider adds tremendous complexity by being extremely flexible and also by including unpredictable muscular efforts at the neck, waist, and hips.

The hope is eventually to understand something of what riders value in terms of the ease or difficulty of balancing and steering. Then, it should be possible to genuinely tune the design to suit a child, a windy-weather commuter, or a racer seeking the most rapid leaning and turning response.

\section{Acknowledgments}

The early phase of this research at Cornell was supported by Andy Ruina's NSF PYI award.

\section{Photo Credits}

Figure 1 courtesy of Andrew Dressel.

Images of the author courtesy of Jim Papadopoulos.

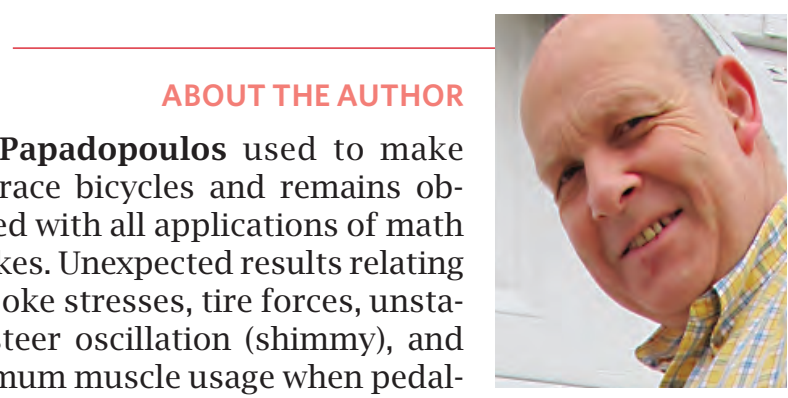
ing may be seen in his co-authored Jim Papadopoulos book Bicycling Science, and others keep turning up. 